\title{
THE IMPORTANCE OF HOLISTIC INTERVENTION IN CHILDREN AND ADOLESCENT OBESITY
}

\author{
Anamaria Gabriela IULIAN ${ }^{1}$, Dorina Maria NIJLOVEANU ${ }^{1}$, \\ Alexandra PREDOIU ${ }^{2 *}$, Radu PREDOIU ${ }^{2}$ \\ ${ }^{1}$ University of Bucharest, Faculty of Psychology and Educational Sciences, Bucharest, Romania \\ ${ }^{2}$ National University of Physical Education and Sport, Faculty of Physical Education and Sport, Bucharest, \\ Romania \\ *Corresponding author: alexandra.predoiu@yahoo.com
}

https://doi.org/10.35189/dpeskj.2020.59.2.8

\begin{abstract}
Adolescence is a special stage in the development of obesity and implicitly for interventions to control it. From a nutritional point of view, the adolescent with weight problems is going through an extremely vulnerable period, the increased need of nutrients necessary for the correct physical and mental development being often unsatisfied due to poor eating habits. This article refers to the importance of sport and physical activity of any kind as an adjuvant treatment of childhood obesity and was conceived as a plea for the holistic approach to infantile obesity. The study briefly presents the pathological substrate of childhood obesity (statistics, causes, consequences, methods of diagnosis and treatment) and the solutions considered most effective in ameliorating and curing this problem. It highlights the role of movement in implementing specific treatments for childhood obesity, along with the modern approach to nutrition in contrast to lifestyle, and also the influence of family patterns on the manifestation of exercise and eating habits and their transmission from one generation to another. Evidence based on empirical research in the literature regarding intervention programmes in the treatment of childhood obesity is also presented. Healthy lifestyle modification programmes for weight control in children should be directed by health professionals (primary health care professionals, nutrition/diet professionals, teachers, physical activity professionals), who are specially trained in the field of infantile obesity.
\end{abstract}

Keywords: childhood obesity, sport and physical activity, nutrition, healthy lifestyle programme.

\section{Introduction}

A recent systematic analysis of 1,769 studies ( $\mathrm{Ng}$ et al., 2014) found that the combined prevalence of childhood overweight and obesity had raised by $47.1 \%$ between 1980 and 2013 worldwide. Yeung (2019) found a global upward trend towards obesity, which was also noted in much of western and southern Europe in the two decades prior to 2000 (Lobstein \& Frelut, 2003).

Childhood overweight and obesity have become a major public health problem in industrialised countries. The definition of childhood obesity must take into account the physiological variations of adiposity during growth and development but also its statistical distribution by age (Creff, 2010). Therefore, the obese child is commonly defined as a child with a body mass index greater than the $97^{\text {th }}$ percentile for age. As there are more and more obese children, interest in this pathology has increased because they have a predisposition to obesity, which will become even higher in adulthood, considering whether they have obese parents as well (Jameson, 2014).

The causes of childhood obesity can be multiple (Creff \& Layani, 2007): 
- Although obesity is not hereditary, some predisposition is currently acknowledged due to better resistance in case of hypocaloric and nutritional deficiencies. Several studies on identical twins raised in different families point out that these children tend to develop weight problems close to those of biological parents; however, the kilocalorie response to a high-fat diet is genetically determined, which is why some individuals show particular sensitivity to obesity and therefore a marked tendency to gain weight (Jameson, 2014). From a pathophysiological point of view, it is difficult to quantify the relevant parameters in energy intake, consumption and storage because of the neuroendocrine and metabolic systems. The adipocyte is an endocrine cell that releases hormones (leptin, adiponectin, resistin), cytokines (TNF- $\alpha$, IL-6), enzymes, complement factors (adipsin), prothrombotic factors (plasminogen activator inhibitor1) etc. The literature describes an intrafamilial aggregation of morbid obesity triggered at a young age, which is explained by inactivation of leptin mutations elevated leptin levels decrease food intake and increase energy intake - or its receptor (LepR) (Jameson, 2014).

- Environmental conditions are determining for the emergence of obesity because there are a variety of foods worldwide with high-energy content, such as fast food or foods rich in sugar and saturated fats, as well as harmful trans fats with a high ability to satisfy the taste of youngsters, and this is a factor that may explain the increased rate of obesity among children (Jameson, 2014). Phillips et al. (2016) highlight that daughters and mothers have almost the same perceptions of eating behaviour, physical activity and health that have been rooted in their daily lives.

- The already mentioned factors are often associated with an increased sedentary lifestyle by spending time in front of different gadgets (laptop, video games, phone or television). Regarding gender, obesity is significantly related to the lack of sports activities only in boys (Glinkowska \& Glinkowski, 2018). Therefore, in modern nutrition, the recommended dietary intakes by age group are calculated not only according to gender and age but especially according to physical activity.

The consequences of childhood obesity are even serious because it is known today that obesity diagnosed before puberty risks remaining a health problem in more than $50 \%$ of the children reaching adulthood (Creff, 2010). Infantile obesity can be complicated by high cholesterol and high blood pressure, with cardiovascular disease in adulthood with a risk of sudden death, myocardial infarction and heart failure, especially in young male adults (Kim et al., 2019). In the case of overweight and obese adolescents, colon cancer neoplasms in adulthood are more commonly diagnosed in women who have suffered from childhood obesity, menstrual disorders, polycystic ovary syndrome, diabetes, joint disease, pregnancy complicated with obesity and gestational diabetes as well as hepatic steatosis. In the last 20 years, there has been much discussion about a new disease diagnosed in children aged 11 to 14, an ultra-early form of non-insulin-deficiency named young type 2 diabetes mellitus (Eschleman, 1996). The treatment of excess weight in childhood obesity is thus imperative from the moment when weight problems have been detected. Childhood overweight and obesity will be treated as soon as possible after the age of 3, especially if one of the parents is obese. A common mistake of doctors in treating overweight and obese patients but also of 
parents is to consider that puberty will regulate the child's weight problems. (Landgraf et al., 2015)

Early treatment of childhood overweight and obesity is supported by a higher success rate in the treatment of children than adults, given that more than a third of adults with weight problems were obese in childhood. The sooner the weight balance is restored, the less severe the metabolic and psycho-emotional consequences will be in adult life, being known that the individual's quality of life is conditioned by a good health condition (Piotrowski \& Kubacka, 2015). Last but not least, it is worth mentioning that, once nutrition education has started in childhood, it is much more likely that the acquired state of health will be maintained in adulthood.

Although the body mass index (BMI) - defined as the ratio of weight in kilograms to height expressed in meters squared - is still considered the best diagnostic and prognostic criterion for children, the rules of modern nutrition are currently guided by body composition (bioelectrical impedance analysis), namely body fat percentage and waist circumference. Thus, numerous studies show an increase in BMI in the first 2 years of life for a normal child, followed by its decrease until around the age of 6 years, and then there is a new increase at the age of 10 based on adiposity rebound; the earlier this adipose rebound occurs, the greater the risk that obesity will persist into adulthood (Landgraf et al., 2015).

\section{Topic addressed}

The treatment of childhood obesity has three main imperatives: correcting an unhealthy eating behaviour, cultivating motivation and positive emotions in children with weight problems as well as "befriending" them with any physical activity. These are the foundations of a healthy lifestyle, which should be adopted by the whole family so that the child with weight problems feels supported, appreciated and helped instead of being punished, restricted and ostracised by scholastic medical rules perceived as difficult to follow. (Hansel, 2013)

Despite these recommendations, certain hygienic-dietary mistakes occur systematically when working with obese children, among which the most common are: a tendency to reject everything that is green or new (neophobia), increased intake of fast carbohydrates (carbonated drinks, biscuits, chocolate, chips, pretzels), which causes dysglycaemia and secondary tachyphagia, excessive sedentary lifestyle (Molin \& Hansel, 2018). Studies have shown that the more children exercise, the easier it will be for them to burn excess fat. It should be noted that, in all sports performed during childhood, games, socialisation and good communication between participants are recommended (therefore, the focus will not be on winning competitions). Among the recommended sports, aerobic exercise is preferred (swimming, running, cycling or rollerblading) over endurance exercise. It is worth mentioning that if, despite all the explanations, a child is not willing to correct their eating behaviour, the prescription of dietary restrictions should be postponed, preferably with an increase in daily energy expenditure based on any type of physical activity practised consistently and lasting at least 30 minutes. There are different ways to encourage children to confidently incorporate movement in their lives and give up sedentary behaviours. One way to ensure success is to reward children every time they adopt movement-related behaviours of any kind. (Carr \& Epstein, 2020) 
A study about the determinants of physical activity and body fat among Chinese adolescents highlights the importance of attitude towards physical activity, screen time, parents' socioeconomic status and exercise habit. Children's intention to participate in physical activities was a strong predictor of their engagement, while parents' exercise habit had both direct and indirect (via attitude) effects on their children's intention to participate in physical activity. Obesity was found to have a negative effect on children's attitude towards physical activity (Yeung et al., 2016). Authors have discussed about the importance of practicing physical activities specific to martial arts, but the organized participation in this sport branch is lower, when compared to other sport disciplines - $2 \%$ girls and $10 \%$ boys (Kudlacek et al., 2019). Childhood obesity has major effects on health and can generate short-, medium- and long-term complications sometimes visible only in adult life. It is linked not only to the increased risk of developing chronic diseases such as diabetes, hypertension, dyslipidaemia, osteoporosis, cardiovascular disease or some cancers but also to psychosocial skills (Landgraf et al., 2015).

Adolescence is a special stage in the development of obesity and implicitly for interventions to control it. From a nutritional point of view, the adolescent with weight problems is going through an extremely vulnerable period, the increased need for nutrients necessary for the correct physical and mental development being often unsatisfied due to incorrect eating habits.

Optimum nutrition and the right supplements are able to increase speed, endurance and strength (Holford, 2005). Aerobic exercise is the optimal physical activity for weight loss. Its advantages are: decreases anxiety and stress by releasing endorphins that give the body and mind a state of peace; reduces the body fat percentage and maintains muscle mass; increases the metabolic rate of rest, which allows more liberal kilocalorie intake; improves muscle tone, which ultimately leads to burning more calories; helps maintain the new bodyweight in the medium and long term (Eschleman, 1996). Optimum nutrition has been shown to increase not only endurance but also sheer muscle power. If referring to proteins, the recommendation for sedentary people is to ingest $0.8-1.0 \mathrm{~g} / \mathrm{kg} /$ day (Kreider, 1999), for endurance athletes, 1.2$1.4 \mathrm{~g} / \mathrm{kg} / \mathrm{day}$, and for people who perform strength training, 1.6-1.7 g/kg/day (Ciocca, 2005). Protein intake immediately after exercise appears to be the optimal solution for anabolism (Phillips, 2004). Regarding creatine, it has beneficial effects on short-term and high-intensity exercise (lasting less than 30 seconds). Performance improvements were also found for longer durations (30-150 seconds) but to a lesser degree. (Branch, 2003) Last but not least, referring to baking soda, it can be used for intense and short-term exercise, improving performance (Gao et al., 1988; Van Montfoort et al., 2004).

Along with genetic and hormonal factors and eating behaviour, the factors that predispose to obesity are a decrease in physical activity or no physical activity at all. Giussani et al. (2013) assessed the role of family history in terms of disease, physical activity and tobacco use to predict the risk of obesity and cardiovascular disease in children and youngsters. The results show that lack of exercise (less than two hours a week), time spent on TV or computer (more than 2 hours a day) and parental smoking are associated with a higher risk of obesity, even if the caloric intake of that child is lower than in an active child.

Physical activity and sedentary lifestyle are included by West and Sanders (2009) as challenges faced by obese children, along with: a) nutrition and b) parental self-efficacy in 
managing adolescent behaviours. Clinical studies show that exercise, physical activity in general promotes fat burning. In order to promote moderate and vigorous physical activity and prevent childhood obesity, Yeung et al. (2016) have underlined the relevance of design interventions that enhance children's intention and attitude towards physical activity by creating customised programmes that take into account their socio-economic status.

In most European countries, the level of physical activity in children is reduced by half until the age of 15, the largest decrease being recorded in Austria, Ireland, Romania and Spain (over 60\%). Research showed that, in 2012, only one in five European children regularly participated in one form or another of sport. Studies show that children's appetite for movement decreases dramatically from the age of 11, especially among girls (Lobstein \& Frelut, 2003). In Romania, there is an increase regarding this problem in children and adolescents, especially in the last two decades (World Health Organization, 2007). Current changes in eating behaviours in terms of quantity and quality, food stereotypes, exercise and sports in relation to media and neuromarketing influences - that promote weight gain - have been considered contemporary features of a modern childhood obesity environment, which creates the premises for a predisposition to weight gain, a significant predictor of obesity in adulthood, with all the corollary specific to a true metabolic syndrome.

Reducing energy expenditure by decreasing physical activity is a factor that predisposes to obesity. The inactivity of obese children is also related to the inactivity of parents. A sedentary lifestyle can be both a cause and an effect of being overweight. The sedentary lifestyle of children and adolescents is due to a multitude of causes. Many communities suffer from a lack of facilities for children to practise physical activity in safety, but the most important factor influencing children's sedentary lifestyles is gadget addiction disorder (Yeung et al., 2016). This disorder has a negative influence on children's eating behaviour, being associated with increased consumption of sweetened beverages, exposure to advertising for unhealthy products and, worst of all, passive overeating (eating in front of the computer or TV without awareness of quantity).

The treatment of childhood overweight and obesity is important, given their significant short- and long-term health and social consequences. The treatment of children is based on the same fundamental principles as the treatment of adults, both aiming to reduce caloric intake through "empty kilocalories" (spoiled by nutritional kilocalories) and increase energy consumption. The main purpose of treatment (weight loss or slowing down weight gain) and the recommended mode of intervention vary depending on the age of the child and the initial level of excess weight (Luttikhuis et al., 2009). The authors conducted a meta-analysis considering studies that reported the use of drug treatment and surgery for the treatment of obesity in children and adolescents. Regarding interventions for the treatment of childhood obesity, this meta-analysis covers: types of interventions, theme of intervention, setting/site of development, delivery method, types of instruments for measuring results, primary and secondary outcomes, and effects of interventions. Based on the types of interventions, the authors identified the following: a) lifestyle interventions (diet, physical activity and/or behavioural interventions); b) drug interventions (orlistat, metformin, sibutramine, rimonabant) and c) surgical interventions (gastric sleeve and gastric by-pass). As for the topics, the authors inventory them as follows: diet and nutrition, physical activity, lifestyle and social support, which involve children themselves with or without associated family 
members. Regarding the delivery method, the authors specify that there are no restrictions, therefore interventions can be applied by researchers, primary health care professionals, nutrition/diet professionals, teachers, physical activity professionals, health promotion agencies, health departments, health or specialist doctors. They identified three types of lifestyle interventions: diet, physical activity and behavioural interventions. Behavioural therapy has been defined as therapy aimed at changing patterns and actions of thinking, especially in terms of food intake and nutrition, physical activity, sedentary lifestyle, food and family environment. According to the mentioned researchers, if the interventions do not specifically include a behavioural programme, they are considered interventions on diet or physical activity. They found nine studies describing activity-based interventions in children under 12 years of age.

Carter and Jansen (2012) note that behavioural interventions for obesity have a very long history, stating that, from a behavioural perspective, the first task is to identify the full range of target eating behaviours involved in obesity on which interventions can be focused. The authors refer to cognitive behavioural therapy (CBT) as a preferred way of treating eating disorders, although they also mention research that reports disappointing results of this type of therapy, in terms of weight loss or weight maintenance. A small number of studies report that cognitive therapy per se may play an effective role in weight loss or weight maintenance. For example, the addition of cognitive therapy to nutritional treatment has been shown to be more effective in relapse prophylaxis compared to the addition of exercise. It has been suggested that cognitive restructuring could have a prophylactic effect on recurrence. Sarvestani et al. (2009), after investigating a multitude of sources and authors, point out that behaviour change techniques (which help people change their physical activity) and healthy behaviours are usually complex, comprising many components that commonly interact. Systematic reviews of the effects of physical activity and healthy eating interventions on behaviour or health outcomes often conclude that both interventions and effect sizes are extremely heterogeneous. While some interventions are indeed highly effective in changing behaviour and health-relevant outcomes, others fail to achieve such effects. Reporting the intervention content is often brief and inaccurate, with interventions generally characterised as, for example, "behavioural counselling", "cognitive behavioural therapy", "motivational strategies" or "practical strategies" used to increase the adoption of "dietary and physical activity recommendations, given that people are concurrently managing family, work and study" and "may have limited finances" (Pettman et al., 2008).

Weihrauch-Blüher et al. (2018) referred to a series of obesity prevention programmes implemented in schools, which aimed at the prophylaxis or reduction of excess weight (primary or secondary prevention), lasting from 4 months to 3 years. The average reduction in BMI was $0.33 \mathrm{~kg} / \mathrm{m}^{2}$. Longer interventions appeared to increase this effect to $0.48 \mathrm{~kg} / \mathrm{m}^{2}$. The therapeutic effect of the interventions was more pronounced in girls compared to boys. The most successful interventions combined physical activity with the optimisation of eating habits.

In Romania, the Protocol for diagnosis and treatment of childhood obesity developed by the "Prof. Dr. Alfred Rusescu" Institute for Mother and Child Protection includes the hygienic-dietary regime, psychological intervention, medication and surgical treatment as parts of the therapeutic intervention. Medication includes: appetite suppressants 
(recommended only for adolescents over 16 years of age, along with diet and short-term physical activity programme) and lipase inhibitors (which cause minimal weight loss). (Dumitru \& Nanu, 2011)

Descriptive explanatory models of childhood obesity support the idea of its holistic, psychological and medical approach. The psychological approach should integrate the perspectives of clinical psychology and psychopathology (focused on psychodynamics and determinant individual mechanisms), social psychology (focused on social practices and sociocultural implications of corporeality) and health psychology (focused on strategies for managing healthy behaviours in the community). The medical literature particularly refers to lifestyle interventions (diet, physical activity and/or behavioural interventions), drug interventions (orlistat, metformin, sibutramine, rimonabant) and surgery (Luttikhuis et al., 2009).

According to Macht (2008), unlike cases of common childhood obesity, obese children diagnosed with eating disorders cannot follow standard treatment (diet correction along with physical activity) because, in compulsive overeating, any imposed food restriction may result in excessive consumption of that food. Another construct involving distress and negative emotions that trigger overeating is diet restriction (Macht, 2008). Attempts to control body weight (e.g. through diet, exercise, appetite suppressants or laxatives) are paradoxically associated with adolescent weight gain. Food restriction is similarly associated with longterm weight gain in female adults. A possible explanation for these contradictions is that people eat excessively in response to a variety of stressful situations. Thus, an individual's mood influences the amount and type of food they choose to eat. Christensen and Brooks (2006) showed that both men and women tended to eat more after a happy versus a negative event. At the same time, the authors pointed out that women were more likely to consume sweets after a sad event compared to men.

According to Blaxter (1990), lifestyle is a popular concept that is often used to describe the choices people make about their consumption patterns; in the health context, these choices involve attitudes towards diet, smoking, alcohol consumption and other health-related habits. Healthy lifestyles are models of self-initiated behaviours and perceptions, which serve to maintain or optimise the quality of life index, with patterns that include aspects such as: hygiene-dietary education, physical activity, nutrition, interpersonal relationships and stress management (Lobel et al., 2008).

\section{Conclusion}

The literature reveals that obesity in adolescents and young adults is linked to an incorrect eating behaviour due to a negative body image and low self-esteem, a decreased level of confidence in physical abilities and a low interest in participating in physical activities. At this time, the generally accepted modern solution in the treatment of childhood obesity is the holistic approach to a family healthy lifestyle. Because a lifestyle change is a slow process, psychotherapy and the support of a group of experts (nutritionists, endocrinologists, psychotherapists, physiotherapists etc.) are always recommended in order to achieve not only weight loss, which is never strictly recommended per se for the child with weight problems, 
but also better intra-family communication, more energy, good mood, increased ability to concentrate and memorise as well as optimal immunity.

Increased physical activity is a mandatory addition to the diet in the right treatment of childhood obesity because it helps prevent some of the side effects of reduced caloric intake, such as a decrease in metabolic rate, more easily regained weight by adipose rebound and loss of muscle tissue. Physical activity contributes to clinical health by promoting better sleep patterns and reducing the risk of chronic diseases such as hypertension, cardiac ischemia, diabetes, metabolic syndrome etc.

In order to be effective, the physical exercise programme should be performed on a regular basis, but the physical education specialist should be consulted before starting an exercise programme.

\section{References}

Blaxter, M. (1990). Health \& lifestyles. London: Routledge. https://doi.org/10.4324/9780203393000

Branch, J. D. (2003). Effect of creatine supplementation on body composition and performance: A meta-analysis. International Journal of Sport Nutrition and Exercise Metabolism, 13(2), 198-226. https://doi.org/10.1123/ijsnem.13.2.198

Carr, K. A., \& Epstein, L. H. (2020). Choice is relative: Reinforcing value of food and activity in obesity treatment. American Psychologist, 75(2), 139-151. https://doi.org/10.1037/amp0000521

Carter, F. A., \& Jansen, A. (2012). Improving psychological treatment for obesity. Which eating behaviours should we target? Appetite, 58(3), 1063-1069. https://doi.org/10.1016/j.appet.2012.01.016

Christensen, L., \& Brooks, A. (2006). Changing food preference as a function of mood. The Journal of Psychology: Interdisciplinary and Applied, 140(4), 293-306. https://doi.org/10.3200/JRLP.140.4.293-306

Ciocca, M. D. (2005). Medication and supplement use by athletes. Clinics in Sports Medicine, 24(3), 719-738. https://doi.org/10.1016/j.csm.2005.03.005

Creff, A. F. (2010). Manual de dietetică în practica medicală curentă [Dietetics manual in current medical practice]. Iași: Polirom.

Creff, A. F., \& Layani, D. (2007). Manuel de diététique en pratique médicale courante [Dietetics manual in current medical practice]. Paris: Masson.

Dumitru, M., \& Nanu, M. (2011). Protocol de diagnostic şi tratament în obezitate la copil [Diagnosis and treatment protocol in childhood obesity]. București: Oscar Print.

Eschleman, M. M. (1996). Introductory nutrition and nutrition therapy. Philadelphia: Lippincott-Raven.

Gao, J., Costill, D. C., Horswill, C. A., \& Park, S. H. (1988). Sodium bicarbonate ingestion improves performance in interval swimming. European Journal of Applied Physiology and Occupational Physiology, 58, 171-174. https://doi.org/10.1007/BF00636622

Giussani, M., Antolini, L., Brambilla, P., Pagani, M., Zuccotti, G., Valsecchi, M. G., Lucini, D., \& Genovesi, S. (2013). Cardiovascular risk assessment in children: Role of physical activity, family history and parental smoking on BMI and blood pressure. Journal of Hypertension, 31(5), 983-992. https://doi.org/10.1097/hjh.0b013e32835f17c7

Glinkowska, B., \& Glinkowski, W. M. (2018). Association of sports and physical activity with obesity among teenagers in Poland. International Journal of Occupational Medicine and Environmental Health, 31(6), 771-782. https://doi.org/10.13075/ijomeh.1896.01170 
Hansel, B. (2013). Perdez votre ventre pour une meilleure santé [Lose your belly fat for better health]. Paris: Hachette Livre.

Holford, P. (2005). The new optimum nutrition bible. New York: Random House Digital, Inc. Jameson, J. L. (2014). Harrison. Endocrinologie [Harrison. Endocrinology]. București: All.

Kim, M. S., Kim, S. Y., \& Kim, J. H. (2019). Secular change in waist circumference and waist-height ratio and optimal cutoff of waist-height ratio for abdominal obesity among Korean children and adolescents over 10 years. Korean Journal of Pediatrics, 62(7), 261268. https://doi.org/10.3345/kjp.2018.07038

Kreider, R. B. (1999). Dietary supplements and the promotion of muscle growth with resistance exercise. Sports Medicine, 27(2), 97-110. https://doi.org/10.2165/00007256-199927020-00003

Kudlacek, M., Groffik, D., Fromel, K., Starsciak, W., \& Stepan, J. (2019). Physical activity in adolescents who prefer and perform martial arts. Archives of Budo, 15, 283-291. http://archbudo.com/view/abstract/id/11940

Landgraf, K., Rockstroh, D., Wagner, I. V., Weise, S., Tauscher, R., Schwartze, J. T., Löffler, D., Bühligen, U., Wojan, M., Till, H., Kratzsch, J., Kiess, W., Blüher, M., \& Körner, J. (2015). Evidence of early alterations in adipose tissue biology and function and its association with obesity-related inflammation and insulin resistance in children. Diabetes, 64(4), 1249-1261. https://doi.org/10.2337/db14-0744

Lobel, M., Cannella, D. L., Graham, J. E., DeVincent, C., Schneider, J., \& Meyer, B. A. (2008). Pregnancy-specific stress, prenatal health behaviors, and birth outcomes. Health Psychology, 27(5), 604-615. https://doi.org/10.1037/a0013242

Lobstein, T., \& Frelut, M. L. (2003). Prevalence of overweight among children in Europe. Obesity Reviews, 4(4), 195-200. https://doi.org/10.1046/j.1467-789x.2003.00116.x

Luttikhuis, H. O., Baur, L., Jansen, H., Shrewsbury, V. A., O’Malley, C., Stolk, R. P., \& Summerbell, C. D. (2009). Interventions for treating obesity in children. Cochrane Database of Systematic Reviews, 21(1). https://doi.org/10.1002/14651858.cd001872.pub2

Macht, M. (2008). How emotions affect eating: A five-way model. Appetite, 50(1), 1-11. https://doi.org/10.1016/j.appet.2007.07.002

Molin, B., \& Hansel, B. (2018). Sans gras ni sucre ou presque! [Without fat, sugar or almost without!]. Paris: Flammarion.

Ng, M., Fleming, T., Robinson, M., Thomson, B., Graetz, N., Margono, C., Mullany, E. C., Biryukov, S., Abbafati, C., Abera, S., F., Abraham, J. P., Abu-Rmeleih, N. M. E., Achoki, T., AlBuhairan, F. S., Alemu, Z. A., Alfonso, R., Ali, M. K., Ali, R., Guzman, N. A., ... Gakidou, E. (2014). Global, regional, and national prevalence of overweight and obesity in children and adults during 1980-2013: A systematic analysis for the Global Burden of Disease Study 2013. The Lancet, 384(9945), 766-781. https://doi.org/10.1016/S0140-6736(14)60460-8

Pettman, T. L., Misan, G., Owen, K., Warren, K., Coates, A. M., Buckley, J. D., \& Howe, P. (2008). Self-management for obesity and cardio-metabolic fitness: Description and evaluation of the lifestyle modification program of a randomised controlled trial. International Journal of Behavioral Nutrition and Physical Activity, 5: 53. https://doi.org/10.1186/1479-5868-5-53

Phillips, E. A., Comeau, D. L., Pisa, P. T., Stein, A. D., \& Norris, S. A. (2016). Perceptions of diet, physical activity, and obesity-related health among black daughter-mother pairs in Soweto, South Africa: A qualitative study. BMC Public Health, 16: 750. https://doi.org/10.1186/s12889-016-3436-8

Phillips, S. M. (2004). Protein requirements and supplementation in strength sports. Nutrition, 20(7-8), 689-695. https://doi.org/10.1016/j.nut.2004.04.009 
Piotrowski, A., \& Kubacka, K. (2015). Soldier's Quality of Life. Journal of Science of the Gen. Tadeusz Kosciuszko Military Academy of Land Forces, 47(1), 97-109. DOI: $10.5604 / 17318157.1158553$

Sarvestani, R. S., Jamalfard, M. H., Kargar, M., Kaveh, M. H., \& Tabatabaee, H. R. (2009). Effect of dietary behaviour modification on anthropometric indices and eating behaviour in obese adolescent girls. Journal of Advanced Nursing, 65(8), 1670-1675. https://doi.org/10.1016/j.nut.2004.04.009

Van Montfoort, M. C. E., Van Dieren, L., Hopkins, W. G., \& Shearman, J. P. (2004). Effects of the ingestion of bicarbonate, citrate, lactate, and chloride on sprint running. Medicine \& Science in Sports \& Exercise, 36(7), 1239-1243. https://doi.org/10.1249/01.mss.0000132378.73975.25

Weihrauch-Blüher, S., Schwarz, P., \& Klusmann, J. H. (2018). Childhood obesity: Increased risk for cardiometabolic disease and cancer in adulthood. Metabolism, 92, 147-152. https://doi.org/10.1016/j.metabol.2018.12.001

West, F., \& Sanders, M. R. (2009). The Lifestyle Behaviour Checklist: A measure of weight- related problem behaviour in obese children. International Journal of Pediatric Obesity, 4(4), 266-273. https://doi.org/10.3109/17477160902811199

World Health Organization. (2007). WHO European Ministerial Conference on Counteracting Obesity: Conference Report. Copenhagen: WHO Regional Office for Europe. https://epdf.pub/who-european-ministerial-conference-on-counteracting-obesityconference-report.html

Yeung, D. C. S. (2019). Correlates of physical activity and obesity in children and adolescents: A meta-analysis and multilevel analysis of Hong Kong Community Fitness Survey. https://scholarworks.iu.edu/dspace/handle/2022/23402

Yeung, D. C. S., Yuan, X., Hui, S. S. C., \& Feresu, S. A. (2016). Determinants of moderate to vigorous physical activity and obesity in children: A structural equation modeling analysis. World Journal of Pediatrics, 12(2), 170-176.

https://doi.org/10.1007/s12519-015-0057-8 